\title{
The effect of electric shock on learning by performers and observers'
}

MILTON E. ROSENBAUM AND OLIVER J. HEWITT

UNIVERSITY OF IOWA

Performers shocked for errors and nonshocked performers explored a multiple-choice bolt-head maze on a first trial. observers were exposed to confederates who replicated the performance of performers on the first trial. On a second (test) trial, observers made fewer errors than performers and performers exposed to shock committed more errors than performers not exposed to shock.

A considerable literature has been assembled on the process of learning by observation of the behavior of others (cf. Bandura, 1965). It is possible to gain further understanding of the variables affecting the observational learning process by comparing the acquisition process of performers with the acquisition process of Ss who observe a performer. Such a comparison can be made by discovering the conditions which differentially affect the acquisition processes of observers versus performers. The present study examines the influence of shock given to a performer for incorrect acquisition responses on the learning of a multiplechoice maze by performers and observers.

Hillix \& Marx (1960) using a multiple-choice learning situation found that Ss who observed, performed better than Ss who were rewarded for their own correct responses. They hypothesized that this unexpected finding was due to interference produced by the error-making activity of performers or to the necessary but nonfunctional act of deciding among responses to make.

In a maze-type task, shocking the performer for incorrect acquisition responses should interfere with learning (Tolman, Hall, \& Bretnall, 1932). Hence a combination of the events suggested by Hillix \& Marx (1960) plus direct punishment for errors should compound the deleterious effects for performers but leave observers relatively unaffected. This last statement should be somewhat qualified in that Berger (1962) has demonstrated that the observation of shock delivery to another has emotional consequences for an observer. Method

A multiple-choice bolt-head maze was employed with 20 rows of four bolt-heads apiece. Above each of the four columns was a letter used for identification purposes. The bolt-heads were arranged in a 4 in, $x 22$ in. rectangle centered on an 18 in. $\times 28$ in. black plywood backboard. On the left-hand margin of the backboard were two jeweled lights. When a performer (P) touched a stylus to a point previously designated as correct, a 6 volt circuit was closed, thereby illuminating a green light. However, when an incorrect point was touched, a red light came on and a relay was triggered which delivered a 90 volt electric shock to a set of electrodes. The duration of this shock, controlled by a Hunter timer, was set at $0.5 \mathrm{sec}$. from the close of the 6 volt relay. The electrodes consisted of sanded-down copper pennies, fastened 1-1/2 in. apart to the inside of a 4 in. wide elastic wrist band. An additional Hunter timer was wired such that a buzzer was sounded every $5 \mathrm{sec}$.

The maze was arranged vertically on a table, $30 \mathrm{in}$. high, directly in front of a seated $\mathrm{S}$. Concealed behind the backboard were the two timers, the shock equipment and all the wiring for the lights.

Each $P$ was seated before the maze while $E$ read the instructions. He was informed that his task was to find and remember the correct point in each of the 20 rows so that on succeeding trials he would commit as few errors as possible. Following the instructions, E placed the electrodes on Ps assigned to the shock conditions (PS) and then turned on the inter-response timer. Ps assigned to the No-shock condition (PNS) were told that no shock would be administered and the electrodes were not attached.

Immediately following the sounding of the buzzer of the inter-response timer, $\mathbf{P}$ touched the stylus to a point in the top row and called out its associated column letter. He then continued through the maze rowby row locating the correct point in one row before moving to the next. Upon completion of all 20 rows, $P$ turned and faced the right-hand wall for a $30 \mathrm{sec}$. rest interval. Prior to the second trial $E$ removed the electrodes, instructing $\mathbf{P}$ that he would receive no more shock.

The procedure for Observers (Os) was similar to the above except that two Ss, one being a confederate, appeared for every session. The confederate, seated to the left of the $S$, moved the stylus on Trial 1 while $O$ called out the column numbers. This focused $O$ 's attention upon the confederate's responses.

In a condition in which $\mathrm{O}$ observed $\mathrm{P}$ receiving shock (OS) the electrodes were attached to the confederate during the first trial. He received a shock of 50 volts to serve as a cue for a mild hand jerk. The electrodes were not attached to the confederate in a condition in which the performer did not receive shock in the presence of an observer (ONS).

As in the $P$ condition, there was a $30 \mathrm{sec}$. delay interval following the confederate's Trial 1 performance. Upon termination of the delay interval $E$ removed the electrodes in the shock condition, announcing that neither $S$ would receive any shock during the remainder of the experiment. In both shock and no-shock conditions the 
Table 1. Mean Errors and Standard Deviations on Trial 1 for Performers and on Trial 2 for Performers and Observers Shock No Shock Total Mean Errors S D Mean Errors S D Mean Errors

\begin{tabular}{lllllll}
\hline Trial 1 & Performers & 27.67 & 2.25 & 26.60 & 1.00 & 27.14 \\
\hline \multirow{2}{*}{ Trial 2 } & Performers & 21.33 & 2.52 & 17.27 & 2.72 & 19.27 \\
& Observers & 14.53 & 3.94 & 12.27 & 2.56 & 13.40 \\
\hline & Total & 17.90 & & 14.76 & & \\
\hline
\end{tabular}

stylus was then handed to $O$ who proceeded to go through the maze for one more trial.

A method was employed which yoked each $O$ to a $P$ by having the confederate follow the response protocol of a previous $P$. The experimenter signalled the appropriate responses to the confederate by placing his hand at different points along the table edge. This signal was blocked from $O$ 's view by the backboard and the side panels; yet it was easily seen by the confederate without moving his eyes from the maze.

The Ss were 60 male undergraduates who were enrolled in an elementary psychology course. Random assignment to the four experimental conditions was employed except that several Ps were run before any Os in order to obtain the necessary first trial response protocols to present to $\mathrm{O}$.

\section{Results}

In Table 1 , the mean number of errors committed by P's on Trial 1 (exploratory) and by Ps and Os on Trial 2 (test) are presented.

On the first trial PS and PNSdid not differ in number of errors $(t=0.52 ; d f=28 ; p>.05)$. A 2 by 2 analysis of variance was conducted on Trial 2 errors. The analysis indicated that significantly more errors were committed in the $P$ than in the $O$ conditions $(F=11.28$; $\mathrm{df}=1 / 56 ; \mathrm{p}<.01$ ) and more errors occurred in the $\mathrm{S}$ conditions than in the NS conditions $(F=3.35 ; p<.07)$ but this difference is short of significant level. Although the interaction term was not significant $(F=1.00)$ the specific hypothesis that more errors should occur in the PS than the PNS condition (Tolman, Hall, \&
Bretnall, 1932) was examined. By t test (one tail) this difference is significant at the 5 percent level.

\section{Discussion}

Along with the findings of Hillix \& Marx (1960) who employed a clearly more complex multiple-choice situation, the present results indicate that Ps suffer interference during the acquisition period that seems not to affect Os. When another factor (electric shock) is introduced to interfere with the performance of $\mathrm{Ps}_{\text {, }}$ this additional event also seems not to affect Os, despite the possibility of vicarious instigation (Berger, 1962).

Unfortunately, the present study offers no further clarification as to the hypotheses concerning error making or decision-making offered by Hillix \& Marx (1960) to account for poorer second trial performance of Ps. In a current study, Os make the decisions and Ps produce the overt responses.

Finally, the capability of O's to profit from observation may be viewed as derived from earlier imitative experience which provides the basis for generalized performance of mediating responses while observing. When given access to the proper manipulandum, the mediating responses are elicited without interfering components, leading to the obtained performance (deCharms \& Rosenbaum, 1960).

\section{References}

Bandura, A. Vicarious processes: A case of no-trial learning. In L. Berkowitz (Ed.), Advances in experimental social psychology. New York: Academic Press, 1965.

Berger, S. M. Conditioning through vicarious instigation. Psychol. Rev., 1962, 69, 450-466.

decharms, R., \& Rosenbaum, M. E. The problem of vicarious experience. In D. Willner (Ed.), Decisions, values, and groups, Vol. I. New York: Pergamon, 1960.

Hillix, W. A., \& Marx, M. H. Response strengthening by information and effect on human learning. J. exp. Psychol., 1960, 60, 97-102.

Tolman, E. C., Hall, C. S., \& Bretnall, E. P. A disproof of the law of effect and a substitution of the laws of em phasis, motivation, and disruption. J. exp. Psychol., 1932, 15, 601-614.

\section{Note}

1. This research was supported by a grant(GS-228) from the National Science Foundation to the senior author. 